\title{
GWAS to the people
}

\author{
Thanks to improvements in data collection and analysis, some polygenic risk scores that predict disease risk are \\ approaching the same predictive accuracy offered by tests for monogenic mutations. The time to think about how \\ best to incorporate polygenic tests in the clinic is now.
}

T he major tenets of genetics are taught in high school by using the example of monogenic disorders, in which an aberration in just one gene is sufficient to increase risk of developing a heritable trait. However, for complex genetic diseases, in which risk is influenced by multiple genetic components along with the environment, the calculus is more intricate. This is wrapped in enough uncertainty that the healthcare field has traditionally shied away from trying to predict an individual's probability of susceptibility to complex genetic disease. Early genome-wide association studies (GWAS) that emerged following the sequencing of the human genome were only able to identify some of the variants that explain the genetic component of complex disease. This limitation made for unreliable calculation of polygenic risk scores (PRSs) in which an individual's probabilistic susceptibility to developing disease is calculated using their genetic variants. It would have been irresponsible to return such scores to individuals and they provide little actionable information. Now, more than a decade later, a study from researchers in Cambridge, Massachusetts, has been able to generate risk scores for common diseases with predictive power in the realm of that achieved for the aforementioned Mendelian diseases (Nat. Genet. https://doi.org/10.1038/s41588018-0183-z, 2018). Pending reproduction of these results and wider clinical testing, healthcare leaders should now begin planning to one day implement PRSs as part of standard of care.

The practice of quantitative genetics, in which phenotypes that vary on a continuum are linked to genetic variants, has provided the groundwork for generating PRSs. This nuanced statistical approach was largely originated in the plant and agricultural world to provide the information required to select livestock and plants for breeding. Its application in human genetics has been a more recent development to understand continuous traits such as height, aided by the development of lab chips used to capture single-nucleotide polymorphisms. However, it has been difficult to calculate reliable PRSs in the clinic for complex disease on the basis of the variants identified by GWAS. This is in part because of sheer numbers: early GWAS typically included only 1,000-5,000 individuals. To be able to identify with any certainty the variants that contribute minimally to a trait, it is necessary for vast numbers of people (both cases and controls) to partake in a study at both the discovery and validation phases. Another previous challenge to generating reliable PRSs was the computational methods available, which lacked power and accuracy. The algorithms required to identify the variants contributing to disease risk have only more recently become sophisticated enough.

Now, the PRSs for coronary artery disease, atrial fibrillation, type 2 diabetes, inflammatory bowel disease and breast cancer have been calculated in populations of European descent. For some individuals, it has been possible to find risk of disease akin to that seen for monogenic disease. The clinical implications of being able to calculate PRSs for disease are well illustrated by cardiovascular disease (CVD). Currently, the best risk factors to calculate whether an individual will develop CVD are lifestyle factors (for example, whether the individual is a smoker), any family history of heart ailments and blood cholesterol levels. Patients may not provide reliable information about the first risk factor and certainly might not be aware of the second. If a PRS indicates that a person has a greater chance of CVD than the general population, this might be enough to push the individual to adopt the recommended changes in lifestyle or for their physician to prescribe statins. It is also important to consider the utility of PRSs not in isolation, but in the context of other tests; for example, in cancer screening, a PRS could be combined with another type of screening such as a mammogram to refine diagnosis.

Whole-genome sequencing has been touted as the next great leap in healthcare, but it is a costly process only the wealthy can afford at present. By comparison, the array chips used in PRS generation are commonly under $\$ 100$, if not much less, making this approach far more affordable. Given the relatively low price point, healthcare providers and hospitals need to consider at what point in life patients should have the option to undergo these tests. In terms of implementing these tests in the clinic, one prior concern has been that most clinicians are not sufficiently experienced to communicate the risk scores generated by the arrays to patients. However, recent preliminary research has shown that providing some training to clinicians in understanding genetic data is sufficient, allowing them to convey to the patients what they mean in terms of risk, whether there are possible treatments or lifestyle changes that would benefit the patient and, if not, the availability of necessary counseling.

The frequency of genomic variants such as those that influence disease reflects population history, and PRSs have historically overlooked under-represented populations. The UK Biobank cohort used in the recent Broad-study, while an amazing resource, over-represents healthy, middleaged people of European descent. As PRS genetic counseling is brought into practice, it will be necessary both to collect data from more varied populations and to consciously apply risk scores with an individual's background in mind. To ensure that this new technology is made available to all, this equity concern needs to be considered in carefully planned clinical trials. The ability to understand one's own health on the basis of genetics must be for everyone.

Published online: 8 October 2018 https://doi.org/10.1038/s41591-018-0231-3 